\title{
A Wireless system for continuous in-mouth pH monitoring
}

\author{
Daryl $\mathrm{Ma}^{* \dagger}$, Christine Mason, Sara S. Ghoreishizadeh*† \\ ${ }^{*}$ Dept. of Electrical \& Electronic Eng., ${ }^{\dagger}$ Centre for Bio-Inspired Technology, Imperial College London, UK \\ Email: \{dm2913, s.ghoreishizadeh14\}@imperial.ac.uk, toothfary.mason@gmail.com
}

\begin{abstract}
An indication of the dental health of patients can be observed from the pH levels of their saliva. This work presents a first prototype of a smart Orthodontic Bracket (SOB) for continuous monitoring of $\mathrm{pH}$ in mouth. The SOB system uses Iridium Oxide (IrOx) $\mathrm{pH}$ sensor with $68.8 \mathrm{mV} / \mathrm{pH}$ measured sensitivity and is powered through Near Field Communications (NFC) using a smart-phone from a distance of $3.5 \mathrm{~cm}$. The system resolves $\mathrm{pH}$ change of 0.15 within a wide range of $\mathrm{pH}$. The system is encapsulated in bio-compatible Epoxy resin and successfully used to measure the $\mathrm{pH}$ in Saliva.

Index Terms-Ph sensor, Near field communication, wireless system, Continuous measurement, wearable system
\end{abstract}

\section{INTRODUCTION}

Saliva sampling is an emerging diagnostic technique used as a non-invasive alternative to blood sampling. Capable of clinical identification of diabetes, inflammation, infections as well as hormonal perturbations [1], it has been increasingly used by researchers and clinicians as part of routine dental and medical office examinations. In particular, the $\mathrm{pH}$ of saliva has been shown to be an indicator of health conditions [2].

The $\mathrm{pH}$ value of saliva in the mouth follows a baseline $\mathrm{pH}$ which varies in the range of [7, 7.5] for a healthy person [3]. 20 minutes after drinking an acidic substance(an example being wine), the $\mathrm{pH}$ level of saliva drops to acidic levels below 5 . This would then revert back to the baseline $\mathrm{pH}$ after approximately 60 minutes. When this baseline $\mathrm{pH}$ occurs at a low level, in the ranges of [5,7], is when there is a danger of the patient contracting tooth decay. This could occur due to teeth enamel issues, or silent gastric reflux. Continuous monitoring and balancing the $\mathrm{pH}$ of the mouth is a essential to reduce bacteria causing tooth decay.

Current commercial options for wireless $\mathrm{pH}$ sensors only consist of large glass electrodes with inbuilt batteries, and are large and cumbersome for wearable usage. Prior research includes the colorimetric detection of the $\mathrm{pH}$ using a smartphone based accessory [4]. Here a test strip containing the users saliva is inserted into an optical system that is integrated into an smart-phone case. The $\mathrm{pH}$ of the saliva is then detected through the application of colour reagents. In [5] an iridium oxide (IrOx) pH sensor was used in tandem with a ResistorInductor-Capacitor (RLC) coil resonator. The $\mathrm{pH}$ variation changes the resonant frequency of the resonator which is then detected using an interrogator coil that transmits power to the circuit at $18 \mathrm{MHz}$.

In this work we present a small wireless and wearable smart orthodontic bracket (SOB) that can be fitted in the mouth and continuously monitor the $\mathrm{pH}$ value of saliva with a high precision. The SOB allows frequent measurement during the day using an Android phone as the reader. This measurement time depends on the reader and would most likely be performed an hour after eating, after having an acidic drink, or just after waking up.

In the next section the design rationale behind the choice of the $\mathrm{pH}$ sensor, energy harvesting and wireless communications are described. Experimental results validating the choices are presented in Section III. In Section IV we show the fabricated prototype and present results of $\mathrm{pH}$ measurement is saliva followed by the conclusion.

\section{System Design}

The SOB device needs to be small to avoid interference with the users jaw motion. Two possible methods for affixing the device exist - either through the use of retainers, or through the use of orthodontic brackets. For a retainer that affixes to the jaw, the shape is slightly more freeform as compared to the bracket method. As the device can extend out towards the side of the mouth, this allows it to avoid the jaw motion completely. Fig. 1 describes the envisaged placement of the SOB on a commonly used retainer and requires a form factor of smaller than $3 \mathrm{X} 3 \mathrm{~cm}$ to ensure safety of the user when placed in the mouth. The two key design choices are the $\mathrm{pH}$ sensing and the energy harvesting module. The component choices behind these two modules are detailed in this section.

\section{A. pH Sensing}

Glass electrodes, Ion Sensitive field effect transistors (ISFET), and $\mathrm{IrOx}$ electrodes are the most commonly used methods for $\mathrm{pH}$ sensing and are compared here based on their sizes and biocompatibility.

Although glass electrodes meet the biocompatibility condition, the smallest commercially available glass electrode are

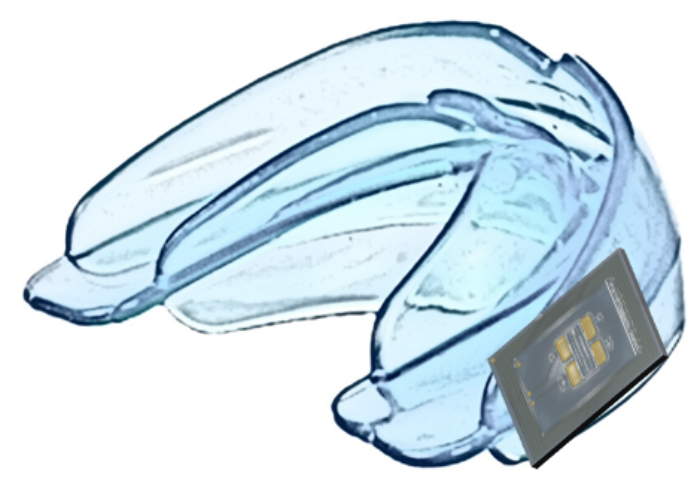

Fig. 1: Envisaged placement of the SOB in commonly used dental equipments 


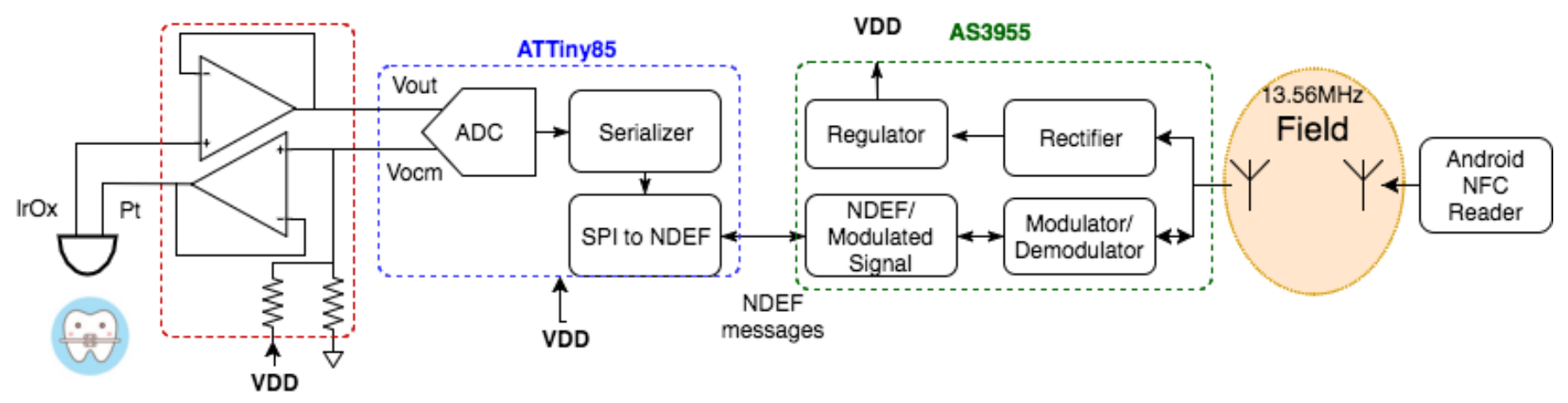

Fig. 2: The block diagram of the proposed $\mathrm{pH}$-monitoring system

more than $10 \mathrm{~cm}$ long and are not suitable in this application due to its large size. ISFETs [6] provide low-cost scalable sensing on unmodified CMOS technology. The ISFET are also passivated in silicon nitride which is bio-compatible. However, they are not yet commercially available and require long fabrication times. IrOx is a bio-compatible material which has been used before in implantable applications [7] and was chosen in this work due to its small form factor and biocompatibility as well as ease of fabrication and relatively high sensitivity to $\mathrm{pH}$.

\section{B. Energy Harvesting}

State-of-the-art physical energy harvesting options such as thermoelectric [8] [9] and motion harvesting [10] [11] [12] provide up to $80 \mu \mathrm{W}$ power and is insufficient to power an embedded system. Inductive coupling was chosen due to the possibility of supplying more than $1 \mathrm{~mW}$ of power. In order to meet The industrial, scientific, and medical radio band (ISM band) requirements, a $13.56 \mathrm{MHz}$-based inductive link was chosen. This also opened up the prospect of using NFC as a means of communication. Commercial NFC ICs were compared, from which the AS3955 IC was chosen due to its small size and standard serial communication such as I2C and SPI as well as EEPROM which makes it capable of storing data to be read from any NFC-enabled device.

\section{Implemented System}

The block diagram of the proposed wireless system is shown in Fig. 2. An open circuit voltage measurement is performed between the IrOx electrode and a platinum wire as the reference electrode. Here a voltage of $V_{D D} / 2$ is applied to the reference electrode through a buffer and the voltage at the IrOx electrode is read through a second buffer that does not allow any current to the sensor. This is done by the analog front-end IC (LMP91200 from TI) which was chosen for its low power consumption $(165 \mu \mathrm{W})$, as well as its internal potential divider that provided a reference voltage. This reduced the need for additional external resistors.

The output of the read out circuit is then fed into an ADC to be digitized. A voltage to $\mathrm{pH}$ conversion is performed

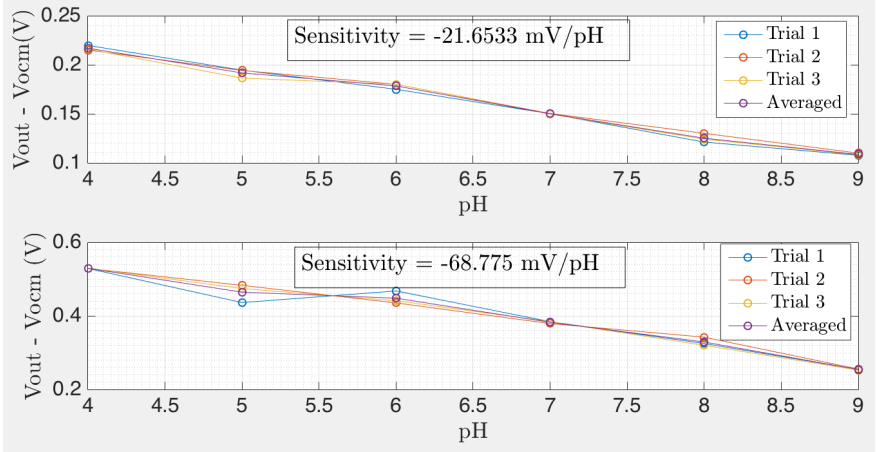

Fig. 3: pH measurement acquired with fabricated IrOx electrode and two different reference electrodes: Pt Wire(top) and $\mathrm{Ag} / \mathrm{Agcl}$ (bottom)

here based on the measured linear characteristics of the $\mathrm{pH}$ sensor. This $\mathrm{pH}$ value is transmitted via NFC Forum Data Format(NDEF) through an SPI interface to the NFC IC. An ATTiny85 microcontroller was used for its low power well as its ability to be configured at SPI interfaces. The clock frequency of the microcontroller is set to a minimum $(128 \mathrm{kHz})$ to consume minimal power.

The received NDEF message at the NFC IC are then converted into a modulated signal (by the NFC Tag Logic available in the AS3955) and is transmitted to the reader (i.e. the smart-phone) via load modulation. The power management unit on the NFC IC consists of a rectifier and a voltage regulator that provide a stable $3.3 \mathrm{~V}$ supply voltage for the microcontroller and the analog front-end IC.

\section{EXPERIMENTAL RESULTS}

\section{A. IrOx pH sensor}

The IrOx sensors were made by oxidizing Iridium wire (178 $\mu \mathrm{m}$ diameter, $99.9 \%$ purity from ADVENT RM) by immersing in solution of Sulfuric Acid (5\% V/V from Sigma). To enable oxidation of $\mathrm{Ir}$, an $\mathrm{Ag} / \mathrm{AgCl}$ reference electrode and a Platinum counter electrode were also placed inside the solution and repetitive Cyclic voltammetries between - 0.2 and 


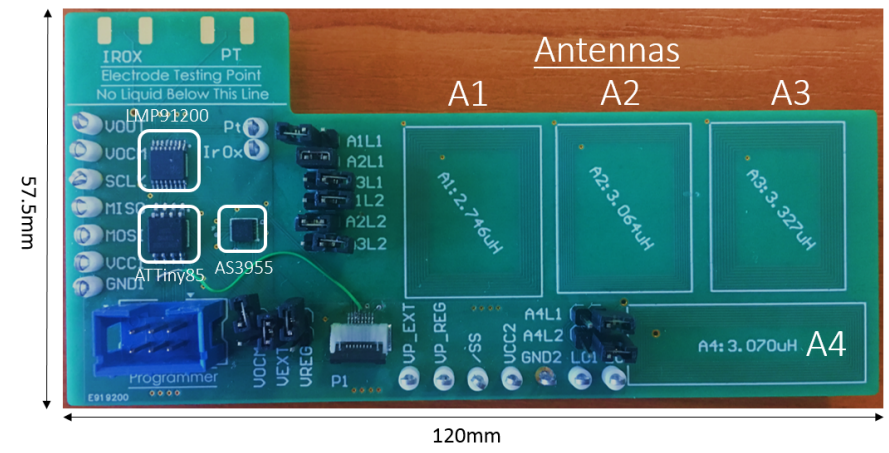

Fig. 4: The photograph of the fabricated test PCB with different antennas designed for testing

\begin{tabular}{c|c|c|c|c}
$\begin{array}{c}\text { Antenna } \\
\text { No. }\end{array}$ & $\begin{array}{c}\text { Simulated } \\
(\mu \mathrm{H})\end{array}$ & $\begin{array}{c}\text { Measured } \\
(\mu \mathrm{H})\end{array}$ & $\begin{array}{c}\text { Max distance* } \\
(\mathrm{mm})\end{array}$ & $\begin{array}{c}\text { Supplied Power } \\
(\mathrm{mW})\end{array}$ \\
\hline A1 & 2.746 & 2.8 & 31 & 4.2 \\
A2 & 3.064 & 3.1 & 35 & 6.3 \\
A3 & 3.327 & 3.3 & 32 & 4.6 \\
A4 & 3.070 & 2.8 & - & - \\
\hline
\end{tabular}

*between antenna and phone case

TABLE I: Antenna Characterization results

$1.2 \mathrm{~V}$ with $1.4 \mathrm{~V} / \mathrm{s}$ scan rate were performed on the three electrode (i.e. Ir wire as the Working electrode) for 3 hours .

A calibration step was run to find the sensitivity of the fabricated $\mathrm{IrOx}$ electrodes using a standard $\mathrm{Ag} / \mathrm{AgCl}$ reference electrodes. The results are plotted in Fig. 3. The average sensitivity was found from three trials to be $-68.775 \mathrm{mV} / \mathrm{pH}$. However, as the standard reference electrodes are quite large, a more ideal reference electrodes in terms of size would be the use of Pt wire. The calibration was repeated with the use of a Pt wire as the reference electrode. The Sensitivity dropped to $-21 \mathrm{mV} / \mathrm{pH}$. This may be due to higher internal resistance of the $\mathrm{Ag} / \mathrm{AgCl}$ electrode [13].

\section{B. Receiving antenna}

To get an $\mathrm{LC}$ resonance at $13.56 \mathrm{MHz}$ when used with the internal $45 \mathrm{pF}$ capacitor of the NFC IC, an inductance of $3.061 \mu$ is required for the antenna. Inspired by the antenna design on the commercial NFC tag, Four rectangular antennas with different geometries (i.e. wire thickness and spacing, diameter) were designed in order to find the optimal design that achieves a maximum output power when exposed to the electromagnetic field at $13.56 \mathrm{MHz}$. The inductance of each antenna, $L_{\text {ant }}$ was first calculated by [14]:

$$
L_{\text {ant }}=2.34 \times \mu_{0} \times N^{2} \times \frac{\frac{d_{\text {out }}+d_{\text {in }}}{2}}{1+2.75\left(\frac{d_{\text {out }}-d_{\text {in }}}{d_{\text {out }}+d_{\text {in }}}\right)}
$$

where $\mathrm{N}$ is the number of turns, $d_{i n}$ and $d_{\text {out }}$ are the inner and outer diameter of the rectangle. An on-line Tool [15] was then used for fine tuning the gap size between the turns and the thickness of the wires and ensure the accuracy of the calculations.
TABLE II: System Performance and design summary

\begin{tabular}{c|c} 
Parameter & value \\
\hline Max distance & $35 \mathrm{~mm}$ \\
\hline pH sensitivity & $-21.6 \mathrm{mV} / \mathrm{pH}$ \\
\hline Min resolvable pH change & 0.15 \\
\hline Encapsulated dimension & $21.01 \times 26.43 \times 5.30 \mathrm{~mm}^{3}$ \\
\hline base material & FR-4 \\
\hline encapsulation & Epoxy resin \\
\hline Power consumption & $3.6 \mathrm{~mW}$ \\
\hline${ }^{*}$ excluding NFC IC &
\end{tabular}

The antennas were fabricated on a PCB (See Fig. 4) and their inductance were measured using a RLC meter. The antennas were then tested together with the other components (NFC IC, microcontroller and the analog font-end) when an smart-phone was used at a controlled distance from it to power on the system. Antennas A1, A2 and A3 were capable of doing so, while antenna $\mathrm{A} 4$, which had a base ground plane, was unable to achieve communications. The maximum distance in air beyond which the connection with the smart-phone reader is lost was measured and listed, together with the power supplied by each of the antennas at that distance in Table I. Antenna A2 shows the ideal properties and was used in the final fabricated prototype.

\section{FABRicATED PROTOTYPe}

The fabricated device can be seen in Fig. 5. It measures $2.1 \mathrm{X} 2.6 \mathrm{~cm}$ (limited by the size of the antenna) and is fabricated (using a commercial PCB technology) on a FR-4 material, at a $1.55 \mathrm{~mm}$ thickness. The measured power consumption of the systems from the generated $V_{D D}$ is $3.6 \mathrm{~mW}$. The three components(AS3955 NFC IC, ATTiny85 microcontroller and LMP91200 analog front-end) are soldered in the center on the top side of the device and the IrOx and Pt electrodes are placed at the bottom part of the PCB together with the antenna. In order to place the device in a user's mouth, or in a liquid buffer for testing, the whole device, except the IrOx sensor and the $\mathrm{Pt}$ wire, is encapsulated in a biocompatible epoxy resin (EPOTEK 301 from Epo-TEK) one side at a time and cured for 2 hours per side. The performance of the SOB is summarized in Table II. The minimum resolvable change is $\mathrm{pH}$ is calculated based on the use of Pt wire as the reference electrode and the 10-bit ADC on the microcontroller.

The encapsulated device is tested with human saliva in order to validate its performance. Different saliva samples are taken at 10 seconds intervals as soda and an acidic meal is consumed. The smart-phone was kept at $2 \mathrm{~cm}$ distance from it. The $\mathrm{pH}$ was read on the smart-phone right away. The results are presented in Fig. 6, showing a clear change in the $\mathrm{pH}$ in both scenarios. The material is consumed at $0 \mathrm{~s}$. The $\mathrm{pH}$ value of the mouth is at $-10 \mathrm{~s}$ indicates the $\mathrm{pH}$ value during before the consumption.

As the soda is consumed, the $\mathrm{pH}$ value detected starts decreasing rapidly. This is due to the high acidity of the soda being detected(pH 3$)$. This acidity is then counteracted by the 
TABLE III: Comparison with state-of-the-art

\begin{tabular}{c|c|c|c|c|c|c|c} 
Paper & pH Sensing & Continuous? & Power cons. & Size & Frequency & Max Distance & Bio. pack. $^{*}$ \\
\hline$[5]$ & IrOx & Yes & Inductive Coupling & $4 \mathrm{~cm} \times 3 \mathrm{~cm}$ & $18 \mathrm{MHz}$ & $18 \mathrm{~cm}$ & No \\
{$[4]$} & Optical & No & Smartphone Battery & NA & - & NA & Yes \\
{$[16]$} & Micro pH Electrode & No & Wired Power Supply & NA & - & NA & Yes \\
This work & IrOx & Yes & Inductive Coupling & $2.1 \times 2.6 \mathrm{~cm}$ & $13.56 \mathrm{MHz}$ & $35 \mathrm{~mm}$ & Yes \\
\hline
\end{tabular}

${ }^{*}$ Bio. pack.= Bio-compatible packaging

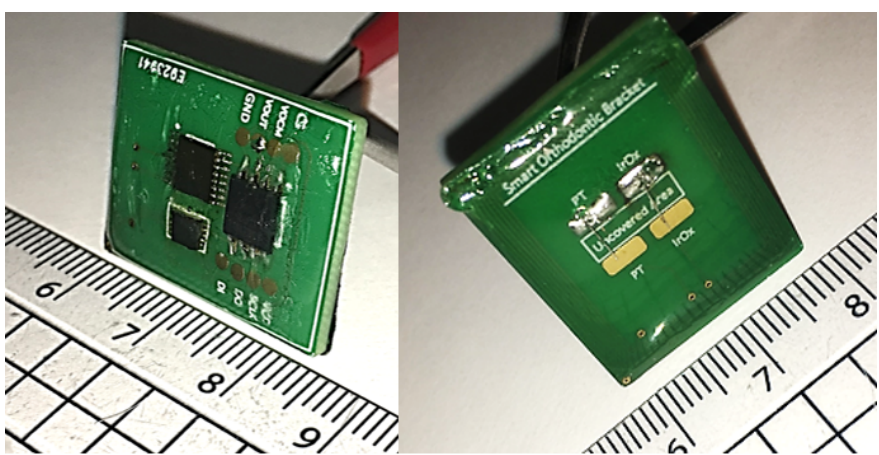

Fig. 5: Final Prototype encapsulated in biocompatible epoxy. Top side with components (left); and bottom side with antenna and $\mathrm{IrOx}$ electrode and $\mathrm{Pt}$ reference electrode.

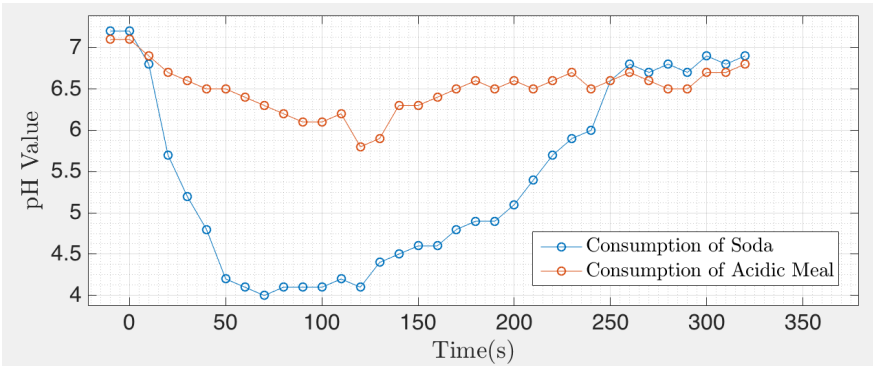

Fig. 6: The measured $\mathrm{pH}$ of saliva using the fabricated prototype, before and after consumption of Soda and meal

mouth producing more saliva, which brings it back up to near the rest state.

A less drastic change in the $\mathrm{pH}$ is observed with the meal. This is due to the meal being solid, which takes time to be broken down before the $\mathrm{pH}$ sensor can begin detection through the saliva. These tests prove the validity of the device as an inmouth $\mathrm{pH}$ monitoring. Table III compares the proposed SOB prototype with state-of-the-art, showing that the presented system is the smallest wearable and wireless device that allows continuous $\mathrm{pH}$ sensing.

\section{CONCLUSION}

A wireless embedded system is presented for continuous $\mathrm{pH}$ sensing in mouth. The system uses standard NFC for power transmissions and data communication. The system consumes $3.6 \mathrm{~mW}$ and is able to perform and communicate to a distance of $35 \mathrm{~mm}$ from a NFC-enabled smart-phone. The final prototype is encapsulated in biocompatible epoxy resin, is relatively small, and has been successfully used to measure the $\mathrm{pH}$ change of saliva after meal and drink consumption.

\section{ACKNOWLEDGEMENT}

The authors would like to thank Prof. Alyssa Apsel for helpful discussion on wireless energy transfer, and Dr. Pantelis Georgiou for his support.

\section{REFERENCES}

[1] G. S. Desai and S. T. Mathews, "Saliva as a non-invasive diagnostic tool for inflammation and insulin-resistance," World J Diabetes, vol. 5, no. 6, pp. 730-738, 2014.

[2] S. Baliga, S. Muglikar, R. Kale et al., "Salivary ph: A diagnostic biomarker," Journal of Indian Society of Periodontology, vol. 17, no. 4, p. 461, 2013.

[3] "How to test your bodys ph (saliva and urine)," 2017. [Online]. Available: https://www.alkaway.com.au/learning-centre/alkalinediet-and-health/how-to-test-your-bodys-ph-saliva-urine/

[4] V. Oncescu, D. O'Dell, and D. Erickson, "Smartphone based health accessory for colorimetric detection of biomarkers in sweat and saliva," Lab on a Chip, vol. 13, no. 16, pp. 3232-3238, 2013.

[5] S. Bhadra, D. S. Tan, D. J. Thomson, M. S. Freund, and G. E. Bridges, "A wireless passive sensor for temperature compensated remote ph monitoring," IEEE Sensors Journal, vol. 13, no. 6, pp. 2428-2436, 2013.

[6] P. Georgiou and C. Toumazou, "ISFET characteristics in cmos and their application to weak inversion operation," Sensors and Actuators B: Chemical, vol. 143, no. 1, pp. 211 - 217, 2009. [Online]. Available: http://www.sciencedirect.com/science/article/pii/S0925400509007059

[7] A. Cavallini, C. Baj-Rossi, S. Ghoreishizadeh, G. D. Micheli, and S. Carrara, "Design, fabrication, and test of a sensor array for perspective biosensing in chronic pathologies," in 2012 IEEE Biomedical Circuits and Systems Conference (BioCAS), Nov 2012, pp. 124-127.

[8] M.-Z. Yang, C.-C. Wu, C.-L. Dai, and W.-J. Tsai, "Energy harvesting thermoelectric generators manufactured using the complementary metal oxide semiconductor process," Sensors, vol. 13, no. 2, pp. 2359-2367, 2013.

[9] M. E. Kiziroglou, S. W. Wright, T. T. Toh, P. D. Mitcheson, T. Becker, and E. M. Yeatman, "Design and fabrication of heat storage thermoelectric harvesting devices," IEEE Transactions on Industrial Electronics, vol. 61, no. 1, pp. 302-309, 2014.

[10] P. D. Mitcheson, "Energy harvesting for human wearable and implantable bio-sensors," in Engineering in Medicine and Biology Society (EMBC), 2010 Annual International Conference of the IEEE. IEEE, 2010, pp. 3432-3436.

[11] J. Olivo, S. Carrara, and G. De Micheli, "Energy harvesting and remote powering for implantable biosensors," IEEE Sensors Journal, vol. 11, no. 7, pp. 1573-1586, 2011.

[12] P. D. Mitcheson, E. M. Yeatman, G. K. Rao, A. S. Holmes, and T. C. Green, "Energy harvesting from human and machine motion for wireless electronic devices," Proceedings of the IEEE, vol. 96, no. 9, pp. 14571486, 2008.

[13] S. Washington, "How a ph meter works," 2017. [Online]. Available: http://www.all-about-ph.com/optical-ph-sensors.html

[14] "An2866 - how to design a 13.56mhz customized tag antenna," 2009. [Online]. Available: http://www.proxmark.org

[15] "NFC design navigator," 2012. [Online]. Available: https://b2bsol.panasonic.biz/semi-spt/apl/en/tool/nfcdesignnavigator/

[16] J. Davidson, R. Linforth, and A. Taylor, "In-mouth measurement of ph and conductivity during eating," Journal of agricultural and food chemistry, vol. 46, no. 12, pp. 5210-5214, 1998. 